BILATERAL COINRACDS

Jerróreen

Sepro Honkapohja

Workins Paper No. 721

WTIONAL BUREAU OF ECONONIC RESHARCH

1050 Massachusetts Avenue

Cambridge M 02138

$$
\text { July } 1981
$$

This research was perforned while Jerry Green was a Fellow of the Center for Advanced Study in the Behavioral Sciences, Palo AIto, California. Support from NSF grant nos. S0C78-06162 and SES80-04815 is gratefully acknowledzed. We would also like to thank Andreu Mas-Colell for helpful discussions and Lynn Gale for performing the computations reported in section 7 . The research reported here is part of the WBEP's research progran in Iconomic Fluctuations. Any opinions expressed are those of the aluthors and not those of the National Bureau of Econonic Research. 


\title{
Bilatera1 Contracts
}

\section{$\underline{\text { ABSTRACT }}$}

The basic form of economic exchange is a bilateral relationship between buyer and seller. If economic conditions are common knowledge there is no problem in principle to determine the efficient quantity to trade. But if benefits are known only to the buyer and costs are known only to the seller a situation of bargaining under incomplete information results. Instead of relying on the vagaries of a bargaining outcome, which might be quite costly to implement, economic inefficiency is likely to be improved by a contractual arrangement that could be agreed upon in advance. In such contracts various aspects of the exchange could be allocated to the two parties involved. For example, a price per unit might be fixed in advance and the buyer might be allowed to name his quantity in the light of the information he has about benefits. A more complex version would present the buyer with a non-linear price schedule. Alternatively the supplier might be given control.

While these solutions are fairly well understood, there are other types of arrangements in which control is mutual. This paper studies contracts of this nature. We examine the feasibility of implementing various agreements and the nature of optimal bilateral contracts under these informational circumstances. When the random influences impact both parties significantly, full efficiency is not attainable. We show that contracts involving mutual control might sometimes be superior to the best contract giving one side or the other exclusive dominance.

\author{
Jerry Green \\ Department of Economics \\ Harvard University \\ (617) 495-4560 \\ Seppo Honkapohja \\ Yrjo Jahnsson Foundation \\ Ludviginkatu 3-5A \\ SF-00130 Helsinki 13 \\ FINLAND
} Cambridge, Massachusetts 02138 


\section{Introduction}

The basic form of economic exchange is a bilateral relationship between buyer and seller. If economic conditions are common knowledge, there is no problem in principle to find the efficient quantity to trade. But if benefits are known only to the buyer and costs are known only to the seller a bargaining situation results. In such circumstances economic efficiency might be improved if a contract governing the transaction could be agreed upon in advance. Such a contract would give control of various aspects of the exchange to the two parties involved. This paper studies contracts of this nature. We examine the feasibility of implementing various agreements and the nature of optimal bilateral contracts.

One approach to this problem is to give control completely to one party or the other. This is seen widely in practice as well as in theory. * A price per unit may be fixed and the buyer can name his quantity after seeing the actual benefits that are relevant. A more complex version presents the buyer with a non-linear price schedule. ${ }^{* *}$ Alternatively, the supplier may be given control in a contract with a specified revenue function along which he can optimize.

These solutions are fairly well understood. When the uncertainty is entirely or primarily on one side of the market they can duplicate the fully efficient solution -- that is the quantity that would be traded in a fullinformation world. When the random influences impact both parties signifi-

\footnotetext{
* Weitzman (1974)

*t Spence (1977)
} 
cantly, full efficiency is not attainable. The choice of which side should govern the contract is then dependent on the elasticity of benefits and costs, and on the distribution of the random parameters.

The primary goal of this paper is to examine contracts that allow for mutual control. While these contracts do not have the ability to achieve the first-best, they may, in some cases, dominate one-sided governance.

In the next section the basic model is set out. It is shorn that feasible contracts lead to traded quantities which, viewed as functions of the random parameters, have to satisfy a certain partial differential equation.

Section 3 examines the special case in which the quantity traded has a certain symmetric dependence on the parameters. This case allows us to restrict the partial differential equation in Section 2, obtaining a secondorder ordinary differential equation. Because this equation is non-linear and because its right-hand side diverges at some points, its solutions divide naturally into several different types. These are studied in Sections 4 and 5, and qualitative properties implied by them for the optimal contract are presented in Section 6.

Numerical methods are used in Section 7 to construct various solutions and verify the theoretical calculations. 
2. The Model

The basic structure of the model follows Weitzman (1974). There is a buyer, whose willingness to pay for the good is

$$
U(q)=\frac{1}{2} a^{2}+\varepsilon q
$$

where $q$ is the quantity traded. The other party to the contract is the seller, whose reservation value for $q$ is the negative of

$$
V(q)=\frac{1}{2} b q^{2}-\delta q
$$

Concavity requires that $a$ and $b$ be negative.

All of the uncertainty in the model enters through the coefficients of the linear terms, $\varepsilon$ and $\delta$. It is useful to note at the outset that the efficient quantity is that which maximizes $U+V$,

$$
q^{*}(\varepsilon, \delta)=-\frac{\varepsilon-\delta}{a+b}
$$

To insure that $\mathrm{q}^{*}$ is positive we suppose that $\varepsilon>\delta$ with probability one. In order to use the methods of incentive compatibility, it is convenient to assume that $(\varepsilon, \delta)$ has a continuous bivariate distribution over a rectangle in $\mathrm{R}^{2}$.

A contract is a pair of functions $t, q$ which assign to each $(\varepsilon, \delta)$ the monetary payment made by the buyer to the seller, $t(\varepsilon, \delta)$, and the quantity received by the buyer from the seller $q(\varepsilon, \delta)$. Given any contract and given the realized values of $\varepsilon$ and $\delta$ the two players can be viewed as participants in a game where the strategies are their professed values of $\varepsilon$ and $\delta, \tilde{\varepsilon}, \tilde{\delta}$ and their payoff functions are respectively 


$$
\mathrm{U}(\mathrm{q}(\tilde{\varepsilon}, \tilde{\delta}))-\mathrm{t}(\tilde{\varepsilon}, \tilde{\delta})
$$

and

$$
\mathrm{V}(\mathrm{q}(\tilde{\varepsilon}, \tilde{\delta}))+\mathrm{t}(\tilde{\varepsilon}, \tilde{\delta})
$$

Viewed in this way, contracts are direct revelation mechanisms in the sense of Green-Laffont (1979) or Laffont-Maskin (1980). We will say that a contract is self-enforcing or incentive compatible if the true value of $\varepsilon$ and the true value of $\delta$ are, respectively, dominant strategies in this game for the buyer and seller respectively. If a contract were not self-enforcing, the value to the two players would have to be computed at the equilibria of the game. Multiple equilibria would typically arise. Little is known about this case. In this paper we examine self-enforcing contracts exclusively.* Moreover, as a technical matter it is convenient to assume that $q(\cdot, \cdot)$ and $t(\cdot, \cdot)$ are twice continuously differentiable.

We now give a characterization of self-enforcing contracts. The optimal strategies for players whose true parameters are $\bar{\varepsilon}$ and $\bar{\delta}$ are determined by the first-order conditions

$$
\text { a } \mathrm{q}(\varepsilon, \delta) \mathrm{q}_{\varepsilon}(\varepsilon, \delta)+\bar{\varepsilon} \mathrm{q}_{\varepsilon}(\varepsilon, \delta)-\mathrm{t}_{\varepsilon}(\varepsilon, \delta)=0
$$

$$
\mathrm{b} q(\varepsilon, \delta) \mathrm{q}_{\delta}(\varepsilon, \delta)-\bar{\delta} \mathrm{q}_{\delta}(\varepsilon, \delta)+\mathrm{t}_{\delta}(\varepsilon, \delta)=0
$$

where subscripts denote partial differentiation. Incentive compatibility requires that these be identities in $(\varepsilon, \delta)$ when evaluated at $\varepsilon=\bar{\varepsilon}$ and $\delta=\bar{\delta}$.

Differentiating ( 6 ) with respect to $\delta$ and $(7)$ with respect to $\varepsilon$ we find (suppressing the arguments of all functions)

*

It may indeed by the case that by using strategy spaces other than the real line, we can implement discontinuous $q(\cdot, \cdot)$ which nevertheless have a lower welfare loss. This is beyond the scope of the present paper. 


$$
\text { a } q_{\varepsilon \delta}+a q_{\delta} q_{\varepsilon}+\varepsilon q_{\varepsilon \delta}-t_{\varepsilon \delta}=0
$$

$$
\text { b q } \mathrm{q}_{\varepsilon \delta}+\mathrm{b}_{\varepsilon} \mathrm{q}_{\delta}-\delta \mathrm{q}_{\varepsilon \delta}+\mathrm{t}_{\delta \varepsilon}=0
$$

Using $t_{\varepsilon \delta}=t_{\delta \varepsilon}$ we can eliminate $t_{\varepsilon \delta}$ from $(8)$ obtaining

$$
((a+b) q+(\varepsilon-\delta)) q_{\varepsilon \delta}+(a+b) q_{\varepsilon} q_{\delta}=0
$$

Equation (10) is the fundamental partial differential equation of this theory of bilateral contracts. In the risk neutral world we are considering, efficiency and welfare losses can be determined entirely by the relationship between $q(\varepsilon, \delta)$ and $q^{*}(\varepsilon, \delta)$. Indeed the form of the benefit and cost functions imply that the expected loss compared with full information is

$$
\frac{1}{(a+b)^{2}} E\left(q-q^{*}\right)^{2}
$$

In any self-enforcing contract the quantity traded must satisfy (10). Optimal contract design can thus be viewed as the problem of minimizing expected welfare losses (11) subject to $(10)$.

Note that any function of only one of the two variables will satisfy (10). This is another way of seeing that one-sided contract governance can be made quite flexible by choosing the non-linear price or revenue functions appropriately.* The first-best given in (3), however, is unattainable through any self-enforcing scheme. ${ }^{* *}$

Second-order conditions for the individuals must be respected as constraints. See below.

$\star \star$

This result is well known, see Green-Laffont (1979). 
Before specializing and examining the nature of the solutions to (10), which will be the subject of the rest of this paper, two further points should be made. The individuals' second-order conditions must hold at each value of the parameters, and this entails some further constraints on the functions $q(\cdot, \cdot)$ that can be implemented. For the buyer, we have that

$$
\mathrm{q}\left(\mathrm{q}_{\varepsilon}\right)^{2}+\mathrm{a} \mathrm{q}_{\varepsilon \varepsilon}+\bar{\varepsilon} \mathrm{q}_{\varepsilon \varepsilon}-\mathrm{t}_{\varepsilon \varepsilon}<0
$$

To express this as a constraint on $q$, note that as (6) is an identity with $\varepsilon=\bar{\varepsilon}$ we can differentiate it with respect to $\varepsilon$. Taking the result and subtracting it from (12) yields

$$
q_{\varepsilon}>0
$$

Similarly, the seller's second-order condition when combined with the firstorder conditions for all $\delta$ yields

$$
\mathrm{q}_{\delta}<0
$$

Finally, the value of the problem for contracts with one-sided governance can be seen from the form of (11). With buyer's control, q should be set at the mean of $\mathrm{q}^{*}$ conditional ${ }^{*}$ on the value of $\varepsilon$. This results in a welfare loss of

$$
\frac{1}{(a+b)} \operatorname{var}(\delta \mid \varepsilon)
$$

$\star$

If the conditional mean of $\delta$ is positively related to $\varepsilon$ with a slope exceeding unity, then the second-order conditions cannot be satisfied in such a solution with buyer's control, and the welfare loss (15) is not actually attainable. We will not discuss this further, as the remainder of the paper deals with two-sided control contracts, but we will be careful to insure the second-order conditions throughout. 
when $\operatorname{var}(\delta \mid \varepsilon)$ is the conditional variance of $\delta$ given $\varepsilon$. And, for sellers' control, the optimum is

$$
\frac{1}{a+b} \operatorname{var}(\varepsilon \mid \delta)
$$




\section{Solutions That Depend on $\varepsilon-6$}

We have shom that any self-enforcing contract entails that the quantity traded satisfy (10). It is thereiore of interest to examine the qualitative characteristics of such solutions in which a is genuinely dependent upon both random shift parameters. We are not actually able to characteriae solutions in general. We focus attention here on those solutions that, like the first-best, depend on $\varepsilon$ and ofhrough their difference

$$
q(\varepsilon, c)=\phi(\varepsilon-\phi)
$$

Our focus on contracts of the form ' 17 ' can be iustifich by an axion which states that equal shifts in the marginal valuations of the buyer and the seller do not alter the quantity traded. Formally this can be written as

$$
q(\varepsilon+h, \varepsilon+h)=q(\varepsilon, b)
$$

for all $(\varepsilon, \delta)$ and $h$ in the donain.*

Under the specification (17) we know that $q_{\varepsilon}=\phi^{\prime}, q_{\delta}=-\phi^{\prime} \cdot q_{\varepsilon \delta}=-\phi^{\prime \prime}$. Thus, defining

논

The assertion is proved as follows. Differentiate (18) to obtain

$$
q_{\varepsilon}+q_{\delta}=0
$$

which we can differentiate again to obtain

$$
q_{E \varepsilon}-q_{\delta \delta}=0
$$
which is the somcalled wave equation in $\mathrm{R}^{1}$ (see, eg., Hellwig (1960), p.11).
Its solutions are of the form.

$$
q(\varepsilon, \delta)=w_{1}(\varepsilon+\delta)+w_{2}(s-\delta),
$$

where $w_{i} \varepsilon c^{2}$ are arbitrary. A direct computation of $q_{\varepsilon}$ and $q_{\delta}$ and a substitution show that $w_{1}=0$ so that the form (17) follows Erom axiom (18). 
(19)

$$
\mathbf{x} \equiv \varepsilon-\delta
$$

$$
\Psi(\mathrm{x}) \equiv \Psi(\varepsilon-\delta) \equiv(\mathrm{a}+\mathrm{b}) \phi(\varepsilon-\delta)+(\varepsilon-\delta)
$$

the basic partial differential equation ( 10 ) takes the form of the ordinary differential equation.

(20) $\quad \Psi^{\prime \prime} \Psi+(1-\Psi)^{2}=0$

The function $\Psi$ has a straightforward economic interpretation:

$$
\Psi=(\mathrm{a}+\mathrm{b})\left(\mathrm{q}-\mathrm{q}^{*}\right)
$$

Therefore, since the optimal contract minimizes $E\left(q-q^{*}\right)^{2}$, we can consider the problem to be

$$
\min \mathrm{E} \Psi^{2}
$$

subject to $\Psi$ solving ( 20$)$. The second-order conditions (13) and (14) impose the constraint

$$
\Psi^{\prime}<1
$$

which, as we will see below, allows us to restrict the class of solutions to (20) corresponding to implementable contracts.

Equation ( 20 ) is an interesting sort of differential equation for several reasons. It has one obvious family of solutions, namely

$$
\Psi(\mathrm{x})=\mathrm{x}+\mathrm{c}
$$


for any real number $c$. In economic terms these are the trivial solutions for, using (19), one can see that they correspond to $q(\varepsilon, \delta)=\frac{c}{a+b}-$ a completely inflexible and uncontingent contract.

There are other solutions to (20), and it is on these that we shall focus. The difficulty in finding some of these solutions can be traced to the fact that it does not define a unique value of $\Psi^{\prime \prime}$ when $\Psi=0$. As is we11-known in the theory of differential equations, the existence and uniqueness of a solution of an equation of order $n$ given $n$ initial conditions is guaranteed in a neighborhood of the initial point only if the equation is Lipschitzian throughout such a neighborhood. The irregularity in this equation occurs at a particularly unfortunate value, $\psi=0$, which is precisely where $q=q *$.

Because of this fact, we will have to discuss solutions other than those given by (24) in two separate cases: those where $\Psi$ has one-sign througout the range of $x$, and those where $\Psi$ is zero for some $x$. These will be called onc-signed and tro-signed contracts respecitvely, and are analyzed scparately in Sections 4 and 5.

We are looking for solutions to (20) over the domain of $\mathrm{x}$ that could possibly arise. It is not necessary that the solution be extendable over the whole real line.* We will see that the solutions other than ( 24 ) indeed have the character that they cannot be extended beyond a bounded interval.

\footnotetext{
Indeed we have already assumed that $(\varepsilon, \delta)$ lies in a rectangle in $\mathrm{R}^{2}$.
} 
Let us consider, at first informally, the qualitative nature of solutions where $\Psi$ has one sign. Suppose we are looking for a solution on $\left[\mathrm{x}_{0}, \mathrm{x}_{1}\right]$ and that we set $\psi^{\prime}\left(\mathrm{x}_{0}\right)<1$, as required by the second-order conditions, and $\Psi\left(\mathrm{x}_{0}\right)>0$. From $(19)$ we can see that $\Psi^{\prime \prime}\left(\mathrm{x}_{0}\right)$ must be negative. Therefore $\Psi^{\prime}$ decreases further with $\mathrm{x}$. At some $\mathrm{x}, \psi^{\prime}=0$ and $\Psi$ is at a maximum; beyond this $\Psi$ begins to decrease. In this region $\psi^{\prime \prime}$ is diverging towards $-\infty$ because $\left(\Psi^{\prime}-1\right)^{2}$ is growing and $\Psi$ is going towards zero.

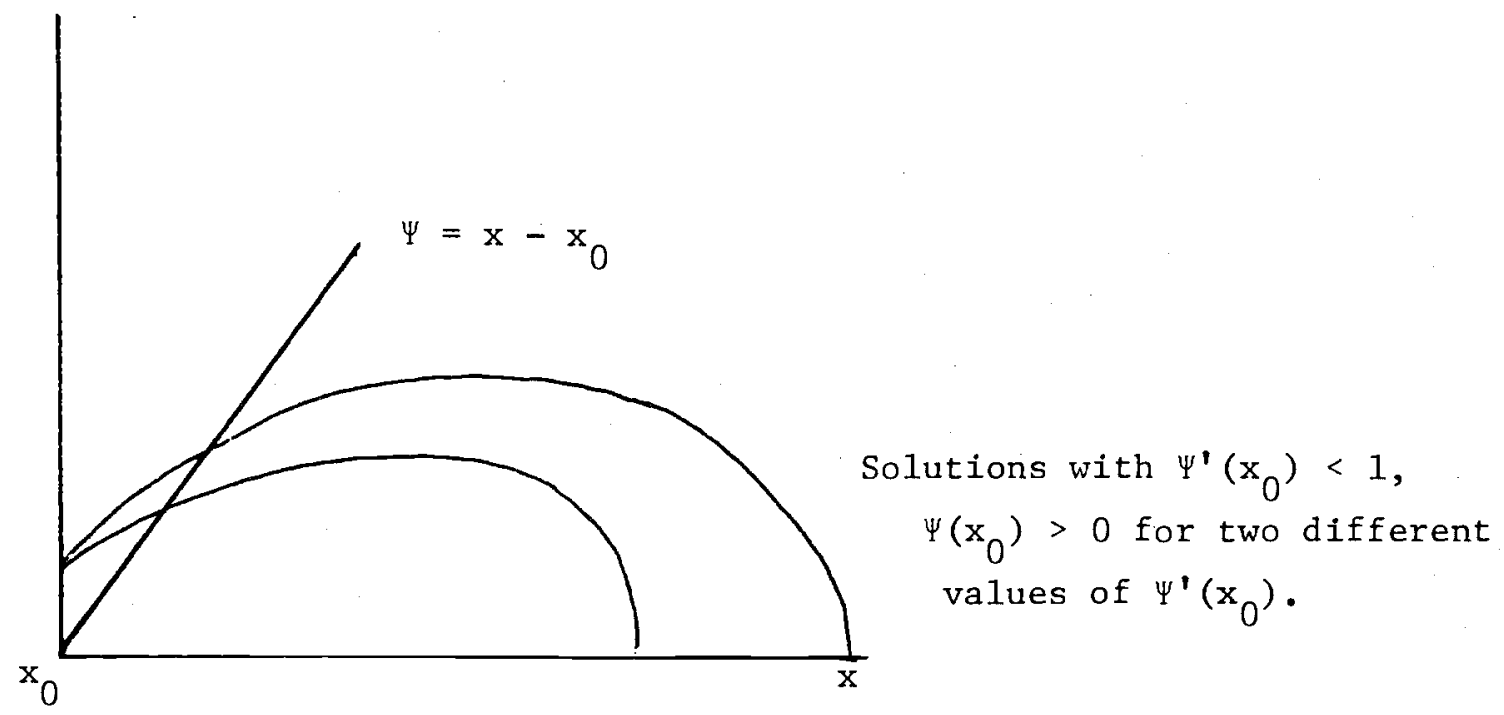

Such a solution exists only on intervals where the upper endpoint is below the point where this degeneracy occurs.

The requirement that the solution exist throughout the range of $\mathrm{x}$ can be viewed as placing constraints on $\Psi^{\prime}\left(\mathrm{x}_{0}\right)$ and $\Psi\left(\mathrm{x}_{0}\right)$. If, for example, the degeneracy were to occur before $x_{1}$, then the value specified for $\Psi\left(x_{0}\right)$ could be increased. It is easy to see that the resulting trajectory would be everywhere higher and would have a degeneracy at a larger value of $x_{1}$. In this way the domain of the solution can be extended. It can also be extended by raising $\psi^{\prime}\left(x_{0}\right)$, which has a qualitatively similar effect. 
4. One Signed-Solutions to $(20)$

In order to obtain manageable expressions for the solution, which will eventually be obtained only by numerical methods, it is useful to convert (20) into a first-order equation. This can be done because (20) is autonomous -- $\mathrm{x}$ does not appear explicitly. Let

$$
z\left(\Psi^{\prime}\right)=\Psi^{\prime}
$$

so that

$$
\frac{\mathrm{d} \Psi^{\prime}}{\mathrm{dx}}=\Psi^{\prime \prime}=\frac{\mathrm{d} z}{\mathrm{~d} \Psi} \frac{\mathrm{d} \Psi}{\mathrm{dx}}=\frac{\mathrm{d} z}{\mathrm{~d} \Psi} \cdot z
$$

Thus (20) can be rewritten as the following first-order equation for $\mathrm{z}$ :

$$
z \frac{\mathrm{d} z}{\mathrm{~d} \Psi} \Psi+(1-z)^{2}=0
$$

This equation is separable in $z$ and $\Psi$ and can be integrated to yield

$$
\log \Psi=\frac{-1}{1-z}-\log (1-z)+K
$$

where $\mathrm{K}$ is a constant of integration.

This can be rewritten using (25)

$$
\Psi=\frac{K}{1-\Psi^{\top}} e^{\frac{-1}{1-\Psi^{\top}}}
$$

Thus (20) has been rewritten as a first-order equation, still autonomous, but rather non-linear in $\psi^{\prime}$. Such equations may be transformed ${ }^{*}$ via differentiation with respect to $\mathrm{x}$

$$
\text { (30) } \quad \Psi^{\prime}=\frac{-K \Psi^{\prime}}{\left(1-\Psi^{\prime}\right)^{3}} \mathrm{e}^{\frac{-1}{1-\Psi^{\prime}}} \Psi^{\prime \prime}
$$


or ${ }^{*}$ using the change of variable

(31)

$$
v(x)=\frac{-1}{1-\psi^{1}}
$$

$$
d v=\frac{-1}{\left(1-\Psi^{p}\right)^{2}} \Psi^{\prime 18} d x
$$

we have

$$
d x=-K v e^{v} d v
$$

Now ( 32 ) can be integrated on the left hand side from $x_{0}$, the lower endpoint of the interval on which we want a solution, up to $x$, and from $v\left(x_{0}\right)$ to $v(x)$ on the right yielding

$$
\left(x-x_{0}\right)=-\left.K(v-1) e^{v}\right|_{v\left(x_{0}\right)} ^{v(x)}
$$

or

$$
x-x_{0}=-K(v(x)-1) e^{v(x)}+k\left(v\left(x_{0}\right)-1\right) e^{v\left(x_{0}\right)}
$$

To simplify this further, we can use the definition of $K$ in terms of $\Psi$ and $\Psi^{\prime},(31)$, together with the definition of $v,(31)$, to write $K$ in terms of $\Psi(\mathrm{x})$ and $\mathrm{v}(\mathrm{x})$ :

$$
K=\Psi(x)\left(1-\Psi^{\prime}(x)\right) e^{\frac{+1}{1-\Psi^{\prime}(x)}}
$$

or

$$
K=-\Psi(x) \frac{1}{v(x)} e^{-v(x)}
$$

Since (36) is an identity in $x$ we can use it twice in (34), evaluated at $x$ and at $\mathrm{x}_{0}$ :

* This transformation involves dividing by $\Psi^{\prime}$, and at $\Psi^{\prime \prime}=0$, this is $i 11-$ defined. However, as $\psi^{\prime}=0$ for only one value of $x$, this is not of consequence when the integration is performed to yield (32), below. 
(37)

$$
\mathrm{x}-\mathrm{x}_{0}=\Psi(\mathrm{x})\left(\frac{\mathrm{v}(\mathrm{x})-1}{\mathrm{v}(\mathrm{x})}\right)-\psi\left(\mathrm{x}_{0}\right)\left(\frac{\mathrm{v}\left(\mathrm{x}_{0}\right)-1}{\mathrm{v}\left(\mathrm{x}_{0}\right)}\right)
$$

or, using the definition of $v$,

$$
\mathrm{x}-\mathrm{x}_{0}=\Psi(\mathrm{x})\left(\Psi^{\prime}(\mathrm{x})-2\right)-\Psi\left(\mathrm{x}_{0}\right)\left(\Psi^{\prime}\left(\mathrm{x}_{0}\right)-2\right)
$$

or, finally,

$$
\Psi^{\prime}(\mathrm{x})=\frac{\mathrm{x}-\left\{\mathrm{x}_{0}+\Psi\left(\mathrm{x}_{0}\right)\left(2-\Psi^{\prime}\left(\mathrm{x}_{0}\right)\right)\right\}}{\Psi(\mathrm{x})}+2
$$

Equation ( 39 ) is a first-order differential equation, linear in $\psi^{\prime}$ although non-linear in $\Psi$ and $x$. Actually, it really describes a family of such equations because $\Psi^{\prime}\left(\mathrm{x}_{0}\right)$ as well as $\Psi\left(\mathrm{x}_{0}\right)$ can be specified arbitrarily.

However, although (39) specifjes the evnlution of $\Psi(\cdot)$ at points $x$ rhere $\Psi(x) \neq 0$, its non-linear solutions cannot be extended beyond a bounded interval. The extent of this domain of $\Psi(\cdot)$ is determined by the choice of $\Psi\left(x_{0}\right)$ and $\Psi^{\prime}\left(\mathrm{x}_{0}\right)$. Consider (34). Since $K>0$ and $v(x)<0$ the first term on the right-hand side is positive. Moreover since for positively signed solutions $v(x)$ converges to zero (from below) as $x$ increases, the first term is decreasing in $\mathrm{x}$. Thus

$$
\mathrm{x}-\mathrm{x}_{0} \leqq \mathrm{~K}\left(1+\left(\mathrm{v}\left(\mathrm{x}_{0}\right)-1\right) \mathrm{e}^{\mathrm{v}\left(\mathrm{x}_{0}\right)}\right)
$$

serves to define the domain of $x$. Using (36) (at $x_{0}$ ) to eliminate $k$ we have from (40) that

$$
\left(x-x_{0}\right) \leqq \Psi\left(x_{0}\right)\left(\Psi^{\prime}\left(x_{0}\right)-2+\left(1-\Psi^{\prime}\left(x_{0}\right)\right) e^{\frac{1}{1-\Psi^{\prime}\left(x_{0}\right)}}\right)
$$

The implication of this restriction on the domain of $\Psi(\cdot)$ for the design of contracts is as follows: Given any joint distribution of $(\varepsilon, \delta)$, we must 
choose $\Psi\left(x_{0}\right)$ and $\Psi^{\prime}\left(x_{0}\right)$ so that $x=\varepsilon-\delta$ satisfies (41) with probability one. This constrains the expression in brackets in (39) and leads to pointwise higher solutions the larger the required domain. 


\section{Two-Signed Solutions to (19)}

In this section it is demonstrated that the entire family of two-signed solutions to (19) obeying $\psi^{\prime} \leqq 1$ can be generated as follows.

Compute a positive one-signed solution on an interval $\left[\mathrm{x}_{1}, \mathrm{x}_{2}\right]$. Extend the solution "backwards" below $x_{1}$, as far as possible -- that is until $\Psi \rightarrow 0$. Call the limit of $x$ for which the right-hand side of (19) is well defined, $x_{0}$. Next, compute a negative one-signed solution over some interval $\left[x_{3}, x_{3}\right]$ and continue the solution beyond $x_{4}$ until $\psi \rightarrow 0$. Let the value of $x$ for which this limit is reached be $x_{5}$. Since (19) is autonomous, one can "shift" this negative solution by $x_{0}-x_{5}$, obtaining a solution over $\left[x_{3}+x_{0}-x_{5}, x_{0}\right)$. Finally, set $\Psi\left(x_{0}\right)=0$. Composing the positive and negative one-signed solutions with this point we have a function defined over $\left[x_{3}-x_{0}-x_{5}, x_{2}\right]$.

We will now show that this function indeed solves (19), in particular at $\mathrm{x}_{0}$, and that all two-signed solutions to (19) are of this form.

To show that such a procedure results in a solution to (19) it is only necessary to show that $\Psi^{\prime \prime}\left(\mathrm{x}_{0}\right)$ exists. By construction (19) will then be satisfied as both terms are zero.

As a preliminary result we will establish that $\Psi^{\prime}(\mathrm{x}) \rightarrow 1$ as $\mathrm{x}^{\prime} \mathrm{x}_{0}$. Reconsider (28)

$$
\log \Psi=\frac{1}{1-\Psi^{\prime}}-\log \left(1-\Psi^{\prime}\right)+\kappa
$$

The trajectories of solutions in the $\left(\Psi, \Psi^{\prime}\right)$ plane are thus parameterized by $\kappa$. We want to show that all trajectories satisfy $\psi^{\prime} \rightarrow 1$ as $\Psi \rightarrow 0$, that is, that they all emanate from the point $\Psi=0, \psi^{\prime}=1$ and not from any point where $\Psi=0, \Psi^{\prime}<1$. 


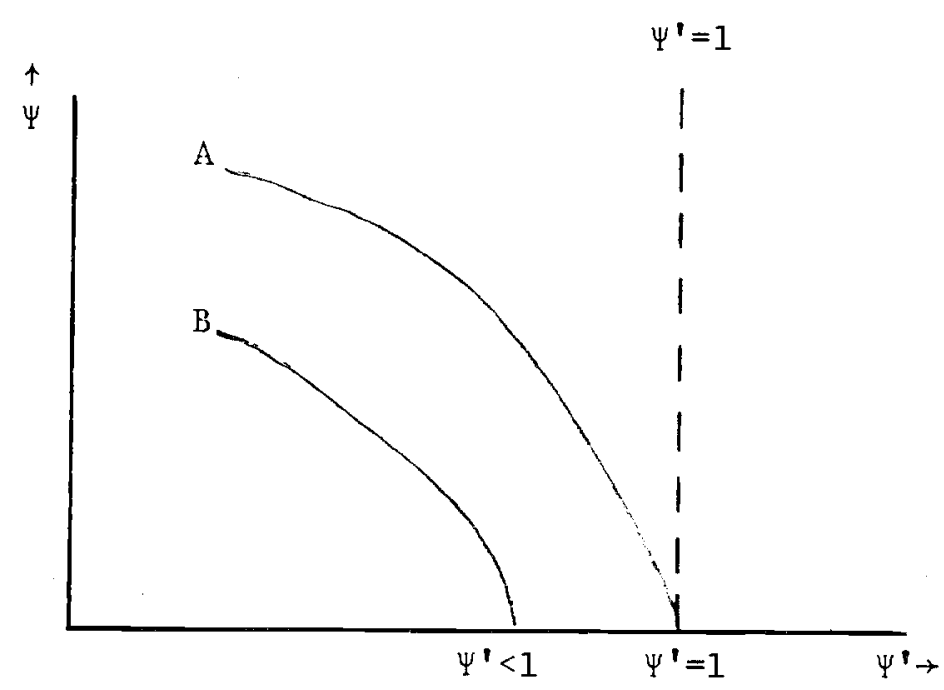

That is that for all $k$ we get a trajectory like $A$ and never like $B$.

If we had one like $B$, then take $\psi^{\prime}>\tilde{\psi}^{\prime}$ and $\psi^{\prime}<1$. For any fixed $\kappa$ and $\Psi$ sufficiently small, the left-hand side of (27) can be adjusted so that it holds. Thus all trajectories cut every vertical line $\left(\Psi^{\prime}<1\right)$ in this plane, and hence $B$ is not a possible trajectory.

Now to show that $\Psi^{\prime \prime}\left(\mathrm{x}_{0}\right)$ exists (and is equal to zero).

At any point in a neighborhood of $x_{0}$ we have

$$
\psi^{\prime \prime}(\mathrm{x})=-\frac{\left(1-\psi^{\prime}(\mathrm{x})\right)^{2}}{\Psi(\mathrm{x})} \text {. }
$$

Using (29) this becomes

$$
\psi^{\prime \prime}(x)=-\frac{\left(1-\psi^{\prime}(x)\right)^{3}}{K} e^{\frac{-1}{1-\Psi^{\prime}(x)}}
$$

From the previous discussion we know that $\Psi^{\prime}(x) \rightarrow 1$ along any trajectory. Therefore

$$
\lim _{x \rightarrow x_{0}} \Psi^{\prime \prime}(x)=0
$$

To show that $\Psi^{\prime \prime}\left(\mathrm{x}_{0}\right)$ exists we apply the mean-value theorem. (Rudin (1964), p. 93 th. 5.10) to the function $\Psi^{\prime}(\cdot)$. We know that $\psi^{\prime}$ is continuous on 
$\left[x_{0}, x_{0}+\eta\right]$ and differentiable on $\left(x_{0}, x_{0}+\eta\right)$ for any $\eta>0$. Thus there exists $0<h(n)<n$ such that

$$
\frac{\psi^{\prime}\left(\mathrm{x}_{0}+n\right)-\psi^{\prime}\left(\mathrm{x}_{0}\right)}{n}=\Psi^{\prime \prime}(\mathrm{h}(n))
$$

Taking limits as $\eta \rightarrow 0$ we find

$$
\Psi^{\prime \prime}\left(\mathrm{x}_{0}\right)=\lim _{n \rightarrow 0} \Psi^{\prime \prime}(h(\eta))=\lim _{x \rightarrow x_{0}} \Psi^{\prime \prime}(\mathrm{x})=0
$$

Precisely analagous arguments apply to the left of $x_{0}$ establishing that $\Psi^{\prime \prime}\left(\mathrm{x}_{0}\right)$ is well defined in this pieced-together function. 


\section{Characteristics of the Payment Function}

In the preceding sections we have analyzed the nature of possible solutions to the fundamental equation (19) derived from (10). These results are important in that they permit a qualitative characterization of the cost function $C(q, \delta)$ of the buyer and the revenue function $R(q, \varepsilon)$ of the seller with respect to the traded quantity $q$.

Given a contract $\mathrm{t}=\mathrm{t}(\varepsilon, \delta), \mathrm{q}=\mathrm{q}(\varepsilon, \delta)$ we can, by utilizing the implicit function theorem, solve the latter to obtain $\varepsilon=\varepsilon(q, \delta)$, because $q_{\varepsilon}>0$ by the second order conditions. Substituting this to the former we obtain the cost function of the buyer $\mathrm{C}(\mathrm{q}, \delta)=t(\varepsilon(q, \delta), \delta]$. By differentiating once

$$
\frac{\partial C(q, \delta)}{\partial q}=t_{\varepsilon} / q_{\varepsilon}
$$

and twice

$$
\frac{\partial^{2} \mathrm{C}(\mathrm{q}, \delta)}{\partial \mathrm{q}^{2}}=\frac{\mathrm{t}_{\varepsilon \varepsilon}}{\left(\mathrm{q}_{\varepsilon}\right)^{2}}+\mathrm{t}_{\varepsilon} \frac{\partial^{2} \varepsilon}{\partial \mathrm{q}^{2}}
$$

To evaluate (47) we have $t_{\varepsilon}$ from (6), $t_{\varepsilon \varepsilon}$ obtained by differentiating (6), and compute

$$
\frac{\partial^{2} \varepsilon}{\partial q^{2}}=\frac{\partial}{\partial q}\left[\frac{1}{q_{\varepsilon}(\varepsilon(q, \delta), \delta)}\right]=-\frac{q_{\varepsilon \varepsilon}}{\left(q_{\varepsilon}\right)^{3}}
$$

In total we have the result

$$
\frac{\partial^{2} C(q, \delta)}{\partial q^{2}}=a+\frac{1}{q_{E}}
$$

the sign of which is in general uncertain. For the special case of solutions dependent on $(\varepsilon-\delta)$ it is possible to rewrite (49) in terms of the function $\Psi$ which was analyzed in the preceding sections. The conclusion is that $t[q, \delta]$ 
is convex (concave resp.) in q whenever $\psi^{\prime}>\left(<\right.$ resp.) $-\frac{b}{a}$.

A similar computation for the seller's revenue function yields the result

$$
\frac{\partial^{2} \mathrm{R}(\mathrm{q}, \xi)}{\partial \mathrm{q}^{2}}=-\mathrm{b}+\frac{1}{\mathrm{q}_{\delta}}
$$

which in turn implies that $t[q ; \varepsilon]$ is convex (concave resp.) in $q$ whenever $\Psi^{\prime}<(>$ resp. $)-\frac{\mathrm{a}}{\mathrm{b}}$.

These results can be interpreted easily by noting that, for example, the convexity of $\mathrm{C}(\mathrm{q}, \delta)$ in $\mathrm{q}$ means that the unit price for the buyer is rising, i.e., quantity premia appear. In the same vein, whenever $c(q, \delta)$ is concave in $q$ the contract stipulates quantity discounts, i.e., the unit price for the buyer is decreasing.

This analysis can be conveniently related to the different types of solution $\Psi$ of the basic differential equation (19). Consider first a positively signed solution discussed in Section 4 above. For then $\Psi^{\prime}$ is first positive, but as $\mathrm{x}(=\varepsilon-\delta)$ increases it turns more and more negative which implies that $R(q, \varepsilon)$ is first convex but it eventually becomes concave. Therefore in these contracts the buyer faces quantity premia at low levels of the traded quantity but discounts appear at high volumes of trading. In Section 7 numerical computations illustrate this phenomenon.

For negatively-signed contracts the conclusion is reversed, i.e., quantity discounts appear at low levels of trading.

As shown in Section 5, two-signed contracts are pieced from one-signed contracts on subintervals so that with them quantity discounts are present at both sufficiently low and high levels of trading while in the intermediate 
range quantity premia are the rule. These features are illustrated in the diagram.

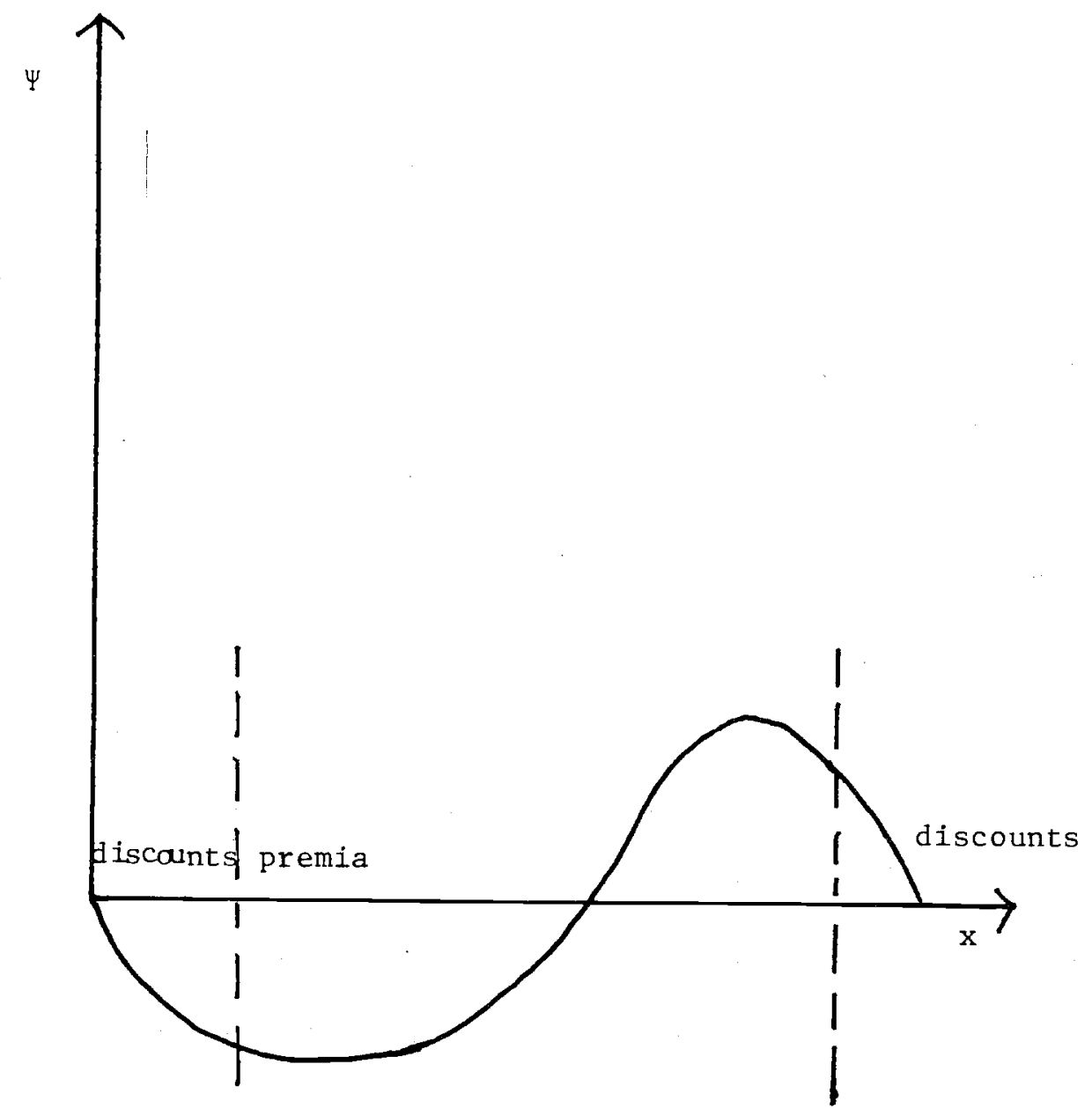

Figure 1 


\section{Numerical Computations}

By virtue of the results in the last three sections a numerical computation of the solutions to (19) can be derived from the one-signed solutions over fixed intervals $\left(x_{0}, x_{\max }\right)$ with the properties that

$$
\lim _{x \rightarrow x_{0}} \Psi=\lim _{x \rightarrow x_{\max }} \Psi=0 .
$$

In this section we examine some qualitative properties of these solutions.

Finding second-best bilateral contracts, that is those which minimize $E \Psi^{2}$, is generally a complex problem. Alternative solutions to (19) cannot be ranked by pointwise dominance. The optimal solution in principal depends heavily on the distribution of $\mathrm{x}=\varepsilon-\delta$.

Despite this theoretical possibility, our computations show that it is relatively easy to find a solution to (19) that is "almost" the pointwise minimizer of $\Psi$ throughout "almost" all of the domain of definition. Therefore, except in extreme cases where the distribution of $\mathrm{x}$ is concentrated close to the endpoints of $\left[x_{0}, x_{\max }\right]$, this particular solution will serve as a good approximation to the optimum.

We now describe the numerical method used and present an illustrative calculation.

Let the length of the interval over which we seek a solution be fixed at $L=x_{\max }-x_{0} \cdot$ From (40) we have an implicit relationship between $\Psi\left(x_{0}\right)$ and $\psi^{\prime}\left(\mathrm{x}_{0}\right)$ that must be satisfied if the solution is to be well-defined over this interval:

$$
\Psi\left(\mathrm{x}_{0}\right) \stackrel{\geq}{=} / \Psi^{\prime}\left(\mathrm{x}_{0}\right)-2+\left(1-\Psi^{\prime}\left(\mathrm{x}_{0}\right)\right) \mathrm{e}^{\frac{1}{1-\Psi^{\prime}\left(\mathrm{x}_{0}\right)}}
$$


Thus for fixed $L$ we consider the one parameter family of solutions to (39) where $\Psi^{\prime}\left(\mathrm{x}_{0}\right)$ is fixed arbitrarily in $(0,1)$ and $\Psi\left(\mathrm{x}_{0}\right)$ is given by the solution to (51) with equality. A standard computer program for numerical integration was used to integrate the expression for $\Psi^{\prime}(\mathrm{x})$ from $\mathrm{x}_{0}$ to $\mathrm{x}$.

Figure 2 displays the results for various choices of $\Psi^{\prime}\left(x_{0}\right)$ when $L=10$. The central feature of this simulation is that the solution obtained for $\Psi^{\prime}\left(\mathrm{x}_{0}\right)=.9$ is "almost" the pointwise minimizer of the family of all solutions obtained for values of $\Psi^{\prime}\left(\mathrm{x}_{0}\right)$ in steps of .02 . Only for $x-x_{0} \leqq .2$ or $x-x_{0} \geq 9.8$ were any of the other solutions below this one.

This result seems robust to the length of the interval and to step sizes of $\psi^{\prime}\left(\mathrm{x}_{0}\right)$ used to construct the families of solutions. It is probably a good approximation to the optimal one-signed solution over this interval for most distributions of $x$.

We then computed the non-linear price and revenue functions that are implicit in the optimal contract, using this result as an approximation for the optimum. This computation was compatible with the results of section 6 on the concavity properties of these functions.

The numerical $\Psi(\cdot)$ obtained above was substituted into the expressions

$$
\begin{aligned}
& t_{\varepsilon}(\varepsilon, \delta)=\frac{(a \Psi+a \delta+b \varepsilon)\left(c_{0}+\psi-(\varepsilon-\delta)\right)}{\Psi(a+b)^{2}} \\
& t_{\delta}(\varepsilon, \delta)=\frac{(b \Psi-b \varepsilon-a \delta)\left(c_{0}+\Psi-(\varepsilon-\delta)\right)}{\Psi(a+b)^{2}}
\end{aligned}
$$

where $c_{0}=x_{0}-\Psi\left(x_{0}\right)\left(2-\Psi^{\prime}\left(x_{0}\right)\right)$. Both a and $b$ were set at -1 . These partial derivatives were integrated numerically over the rectangle $(\varepsilon, \delta) \varepsilon([5,10] \times[0,5])$ so that, as required, $\varepsilon-\delta \varepsilon[0,10]$. The value of $t(5,0)=0$ was taken as a 
normalization without loss of generality.

From this function $t(\varepsilon, \delta)$ we computed the price and revenue functions as follows. For each value of $\delta$ and each $q \varepsilon\left(\frac{5-\delta}{2}, \frac{10-\delta}{2}\right)$ we find the value of $\varepsilon$ so that $\frac{-1}{2}(\Psi(\varepsilon-\delta)-\varepsilon-\delta)=q$. This gives us a function $\varepsilon(q, \delta)$ whose interpretation is that it is the "announcement" of $\varepsilon$ which, when combined with the given value of $\delta$, would induce the given value of $q$ to be exchanged under the contract $\Psi$. Finally, the total cost to the buyer of quantity $C(q, \delta)$, is defined by $C(q, \delta)=t(\varepsilon(q, \delta), \delta)$. The revenue functions of the seller facing a buyer whose announced parameter is $\varepsilon, R(q, \varepsilon)$, is given symmetrically.

Some of these cost and revenue functions are given in Figures 3 and 4 . It is noteworthy that the quantity discount/quantity premia results of Section 6 are verified in this numerical construction.

Because we cannot find the true optimum without knowing the distribution of $x$, and, more importantly, because we cannot find general incentive compatible contracts other than those in the special case $q(\varepsilon, \delta)=\phi(\varepsilon-\delta)$, these numerical results should be viewed as merely illustrative. 

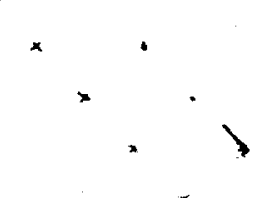

$\alpha$
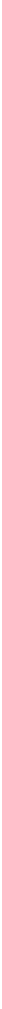


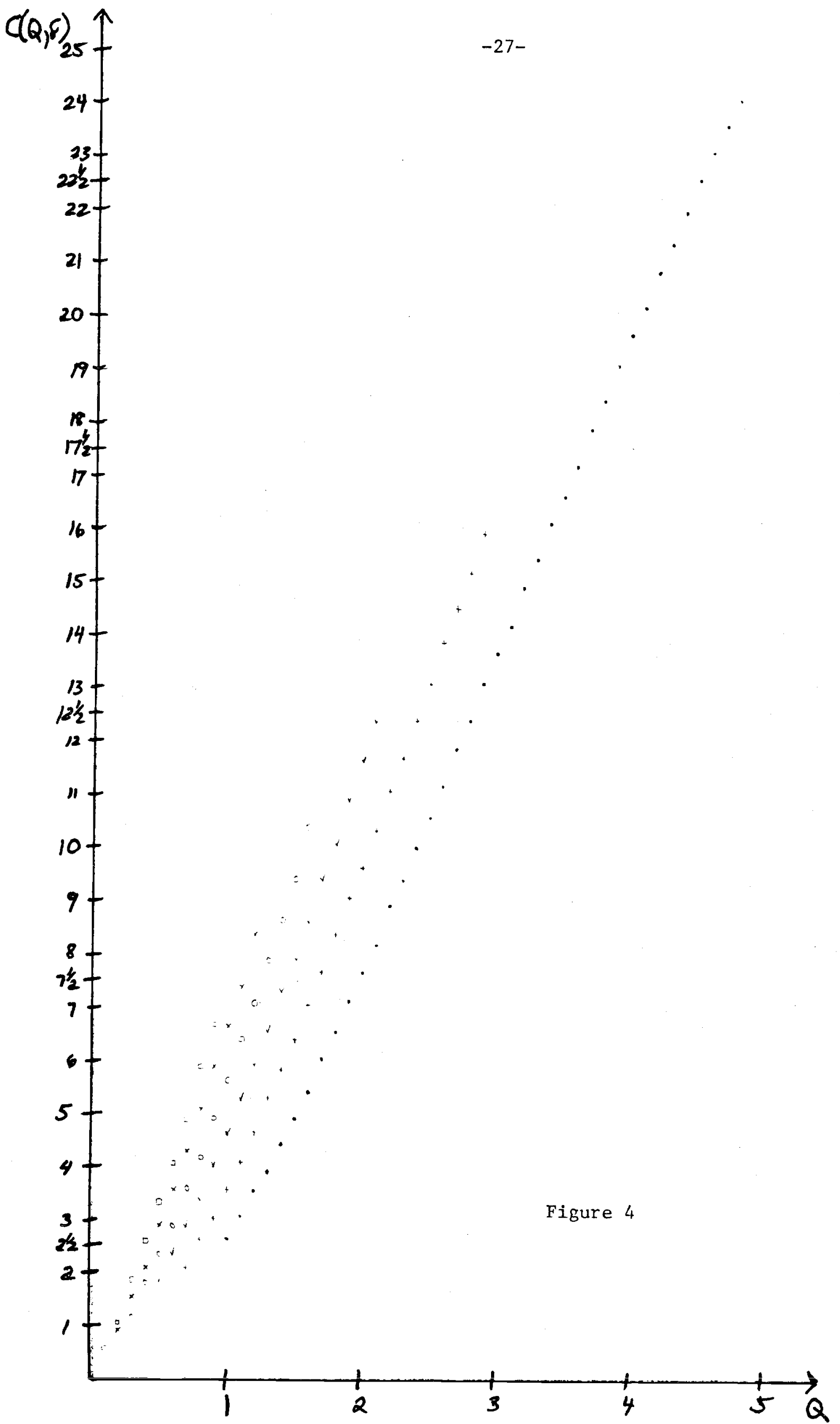


$\underline{\text { References }}$

Ames, W. F. (1968), Non-linear Ordinary Differential Equations in Transport Processes, Academic Press.

Green, J. and J.-J. Laffont (1979), Incentives in Public Decision Making, North-Holland Publishing Company.

Hellwig, M. (1960), Partial Differential Equations, Blaisdell Publishing Co., New York.

Laffont, J.-J. and E. Maskin (1980), "A Differential Approach to Dominant Strategy Mechanisms," Econometrica, vol. 48, 1507-1520.

Rudin, W. (1964), Principles of Mathematical Analysis, McGraw-Hill, N.Y., 2nd ed.

Spence, A. M. (1977), "Non-linear Prices and Welfare," Journal of Public Economics, vol. 8, August, pp. 1-18.

Weitzman, M. (1974), "Prices vs. Quantities," Review of Economic Studies. 